monitoring activation-state markers, such as phosphorylated proteins, and the study of disease progression.

\section{Read-out}

Biosensors for tagging proteins to monitor their activation levels and distribution continue to get more sensitive and versatile. The impact of green fluorescence protein (GFP) and its variants in studying cell signalling is immense. Biosensors that use GFP can, for example, detect conformational changes in proteins in response to ligand binding, changes in protein localization or changes in protein activity.

New fluorescent dyes are also being developed. Klaus Hahn and his colleagues at the University of North Carolina School of Medicine, Chapel Hill, recently reported a biosensor that can visualize the natural dynamics of an unlabelled endogenous intracellular signalling protein, the GTPase Cdc42, in living cells. The sensor is composed of the Cdc42binding fragment of the Wiskott-Aldrich syndrome protein (WASP), to which a novel merocyanine dye has been coupled. The dye is sensitive to changes in hydrophobicity that occur at the interface between the interacting proteins. Binding of WASP to GTP-activated $\mathrm{Cdc} 42$ causes the dye to fluoresce. The sensitivity provided by direct excitation of a novel fluorescent dye enables detection of protein activation at native levels.

One of the more novel detection platforms is quantum dots (see 'Quantum dots show their true colours', page 247); another is

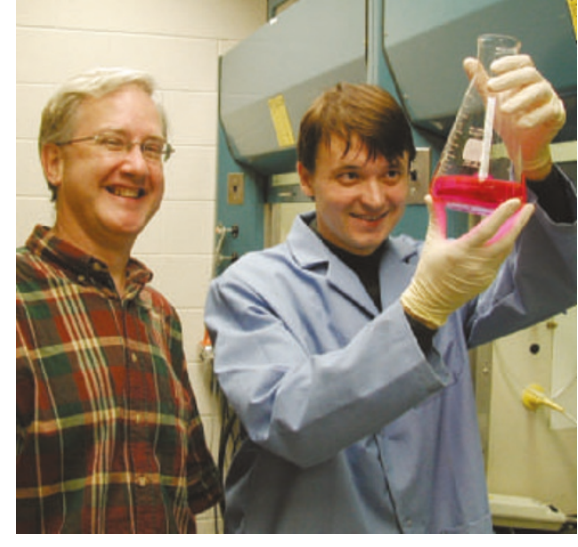

Lighting-up protein interactions:

Klaus Hahn (left) and Alexei

Toutchkine with the dye on which their biosensor is based.

single-molecule photon stamping spectroscopy, which is being used to study the dynamics of the interactions of single proteins (see 'Probing real-time protein interactions').

LI-COR Biosciences of Lincoln, Nebraska, offers two-colour near-infrared fluorescence detection of signal transduction events. The firm originally applied its infrared detection technology to western blots, "but a western blot is not particularly convenient for looking at a pathway", says Michael Olive, LI-COR's vice-president of research and development. The company has developed the In-Cell Western assay for quantifying proteins in fixed cultured cells in 96- or 384-well microplates in less time than conventional western blots by bypassing the need for lysate preparation and the use of gels and membranes. The use of two spectrally distinct near-infrared dyes effectively doubles the number of endpoints that can be analysed, enabling, for example, the measurement of both phosphorylated extracellular signal-regulated kinases (ERKs) and total ERK protein at the same time.

With slight tweaking, the 'In-Cell Western' can become the 'On-Cell Western'. This was developed by James Wager-Miller in the department of anaesthesiology at the University of Washington in Seattle, who is using it to follow the internalization and recycling of GPCRs, in particular the cannabinoid receptor 1 , to and from the cell surface. The hope is that this will lead to a better understanding of how these trafficking events can lead to the desensitization of cells following prolonged or repeated exposure to agonists.

The In-Cell Western is amenable to automation and this month the company will launch a new two-colour plate reader called Aerius, which automates the assay. This may take the technology "into the realm of lead validation", says Olive, helping to prevent costly drug failures later on.

The next challenge for cell signalling will be to look at cellular behaviour on a global scale and for this further improvements in technology will be needed, along with better computational and mathematical tools for deriving information about complete signal-transduction networks.

Diane Gershon is assistant editor, Nature Medicine Technical Reports.

\title{
PROBING REAL-TIME PROTEIN INTERACTIONS
}

Interactions between proteins are one of the main ways in which signals are transferred onward in intracellular signalling pathways. But few methods of studying these interactions can capture the dynamic nature of molecular recognition within signalling complexes.

A group led by Peter Lu, senior research scientist in the W.R. Wiley Environmental Molecular Sciences Laboratory at Pacific Northwest National Laboratory in Richland, Washington, has now developed a method to do just that.

Using a technique called single-molecule photon stamping spectroscopy, which detects and analyses photons that are emitted as single proteins interact, the group has been able to capture proteins in motion as they flip-flop against each other in a manner somewhat analogous to line flicking in fly-fishing. "This type of behaviour cannot be measured by static structure measurements such as X-ray crystallography or NMR," says Lu.

His team has used this approach to study the interaction of single molecules of activated Cdc42 with the dye-labelled WASP (Wiskott-Aldrich syndrome protein) biosensor developed in collaboration with Klaus Hahn and his colleagues (see main text above). Their results indicate highly dynamic — rather than static — interactions between this pair of signalling proteins.

Lu's set-up is largely 'home-built'. Measurements of the interaction of single molecules of Cdc42 with the dye-tagged WASP fragment are made using confocal fluorescence microscopy with laser excitation.



Fluorescence photons are directed towards a photon-stamping detector, which records the intensity and duration of the photon peaks; the data are then analysed to determine the dynamics of the protein-protein interaction.

Technical limitations at present are photobleaching of the dye molecule by the excitation laser and movement of the complex away from the laser focal point. Lu is experimenting with methods for confining the complex either using agarose gel or by tethering one of the protein partners to a glass surface.

Lu plans to extend the single-molecule spectroscopy approach to other important biomolecular complexes under physiological conditions, ultimately hoping to study singlemolecule protein conformational dynamics in living cells. "Right now, the most interesting part may not be the technique but the new scientific information we learn," he says. 